MATHEMATICS OF COMPUTATION

Volume 68, Number 227, Pages 1109-1120

S 0025-5718(99)01070-4

Article electronically published on February 19, 1999

\title{
EXPONENTIAL CONVERGENCE OF A LINEAR RATIONAL INTERPOLANT BETWEEN TRANSFORMED CHEBYSHEV POINTS
}

\author{
RICHARD BALTENSPERGER, JEAN-PAUL BERRUT, AND BENJAMIN NOËL
}

\begin{abstract}
In 1988 the second author presented experimentally well-conditioned linear rational functions for global interpolation. We give here arrays of nodes for which one of these interpolants converges exponentially for analytic functions
\end{abstract}

\section{INTRODUCTION}

Let $f$ be a complex function defined on an interval $I$ of the real axis and let $x_{0}, x_{1}, \ldots, x_{n}$ be $n+1$ distinct points of $I$, which we do not assume equidistant or ordered. Let $f_{k}:=f\left(x_{k}\right), k=0(1) n$. Then

$$
P_{n}[f](x):=\sum_{k=0}^{n} f_{k} L_{k}(x), \quad L_{k}(x):=\prod_{j=0, j \neq k}^{n} \frac{x-x_{j}}{x_{k}-x_{j}},
$$

is the Lagrangian representation of the unique polynomial of degree at most $n$ interpolating $f$ between the points $x_{k}, k=0(1) n$.

Introducing the notations [Sch]

$$
\lambda_{k}:=\frac{1}{\prod_{j \neq k}\left(x_{k}-x_{j}\right)}, \quad k=0(1) n,
$$

and

$$
L(x):=\left(x-x_{0}\right)\left(x-x_{1}\right) \cdots\left(x-x_{n}\right),
$$

we can rewrite (1) as

$$
P_{n}[f](x)=L(x) \sum_{k=0}^{n} \frac{\lambda_{k}}{x-x_{k}} f_{k} .
$$

$P_{n}[f]$ can also be written in its barycentric form by making use of the relation

$$
1=L(x) \sum_{k=0}^{n} \frac{\lambda_{k}}{x-x_{k}} \text {. }
$$

Received by the editor February 10, 1998.

1991 Mathematics Subject Classification. Primary 65D05, 41A20, 41A25.

Key words and phrases. Interpolation, rational interpolation, linear interpolation, exponential convergence. 
Indeed, dividing (4) by (5) we obtain

$$
P_{n}[f](x)=\frac{\sum_{k=0}^{n} \frac{\lambda_{k}}{x-x_{k}} f_{k}}{\sum_{k=0}^{n} \frac{\lambda_{k}}{x-x_{k}}},
$$

which is one of the most stable formulas for evaluating $P_{n}[f]$ [Hen, Ber1].

For several arrays of points the $\lambda_{k}$ (which are called weights of the barycentric formula) can be simplified. For example, for the Chebyshev points of the first kind

$$
x_{k}:=\cos \left(\phi_{k}\right), \quad \phi_{k}:=\frac{2 k+1}{n+1} \frac{\pi}{2}, \quad k=0(1) n,
$$

$P_{n}[f]$ becomes

$$
P_{n}[f](x)=\frac{\sum_{k=0}^{n} \frac{(-1)^{k} \sin \left(\phi_{k}\right)}{x-x_{k}} f_{k}}{\sum_{k=0}^{n} \frac{(-1)^{k} \sin \left(\phi_{k}\right)}{x-x_{k}}} .
$$

And for the Chebyshev points of the second kind

$$
x_{k}:=\cos \left(\phi_{k}\right), \quad \phi_{k}:=\frac{k \pi}{n}, \quad k=0(1) n,
$$

one has

$$
\lambda_{k}=\frac{2^{n-1}}{n}(-1)^{k} \delta_{k}, \quad k=0(1) n
$$

with

$$
\delta_{k}:= \begin{cases}1 / 2, & x_{k}=-1 \text { or } x_{k}=1, \\ 1, & \text { otherwise, }\end{cases}
$$

which gives the remarkably simple formula of Salzer [Sal]

$$
P_{n}[f](x)=\frac{\sum_{k=0}^{n} \frac{(-1)^{k}}{x-x_{k}} f_{k}}{\sum_{k=0}^{n} \frac{(-1)^{k}}{x-x_{k}}},
$$

where the " means that the first and the last terms of the sum are to be multiplied by $1 / 2$.

Global rational interpolation is known to give better results than polynomial interpolation in some examples. But the traditional rational interpolation problem (find a rational function $R_{n}[f]$ with the same number of coefficients as the number of interpolation points) has two major flaws:

- In some cases the interpolation condition $R_{n}[f]\left(x_{k}\right)=f\left(x_{k}\right)$ cannot be satisfied: So-called "unattainable points" occur [Sto].

- $R_{n}[f]$ can have poles in the interval of interpolation, and those poles make the interpolation with $R_{n}[f]$ useless if $f$ is not singular at the same points. 
In Section 1, we recall the rational function proposed in [Ber1] and we derive it in an alternate way by interpolating two functions of two variables. In Section 2, we prove its exponential convergence when interpolating between conformally transformed Chebyshev points. This result is confirmed by numerical experiments (Section 3), which also demonstrate that such a shift of the Chebyshev points often improves the precision of the calculated derivatives as compared with those of the polynomial interpolating between Chebyshev points.

\section{A linear rational interpolant}

Let us denote by $E_{n+1}:=\left\{x_{k}: k=0(1) n\right\}$ a set of distinct points in the interval $[-1,1]$ and define the numbers

$$
\eta_{k}:= \begin{cases}\sqrt{1-x_{k}^{2}}, & -1 \notin E_{n+1}, 1 \notin E_{n+1}, \\ \sqrt{\frac{1}{2}\left(1+x_{k}^{2}\right)}, & -1 \notin E_{n+1}, 1 \in E_{n+1}, \\ \sqrt{\frac{1}{2}\left(1-x_{k}^{2}\right)}, & -1 \in E_{n+1}, 1 \notin E_{n+1}, \\ 1, & -1 \in E_{n+1}, 1 \in E_{n+1} .\end{cases}
$$

In [Ber1] the second author proposed a new, experimentally well-conditioned linear rational interpolant without poles in the interval of interpolation.

Theorem 1. The rational function

$$
R_{n}[f](x):=\frac{\sum_{k=0}^{n} \frac{(-1)^{k} \delta_{k} \eta_{k}}{x-x_{k}} f_{k}}{\sum_{k=0}^{n} \frac{(-1)^{k} \delta_{k} \eta_{k}}{x-x_{k}}}
$$

where $f_{k}:=f\left(x_{k}\right)$ and where the $\delta_{k}$ and the $\eta_{k}$ are given in the formulas (11) and (13), interpolates $f$ between the points $x_{k}, k=0(1) n$, and does not have any pole in the interval $[-1,1]$.

Notice that, according to (8) and (10), $R_{n}[f]$ coincides with the interpolating polynomial when the $x_{k}$ 's are Chebyshev points (see [Ber1]).

1.1. Study of the rational interpolant. In the present subsection we reconstruct the rational function (14) as the quotient of two interpolants, using the same technique as in [Ber2].

Let $I, J$ be two intervals in $\mathbb{R}$, let $g$ be a conformal map from a domain $\mathcal{D}_{1}$ in $\mathbb{C}$ containing $J$ to another domain $\mathcal{D}_{2}$ containing $I$ and such that $g(J)=I$, and let $f$ be a complex function defined on the interval $I$. Without loss of generality, set $J=[-1,1]$.

We define $x_{k}:=g\left(y_{k}\right)$, where the $y_{k}, k=0(1) n$, are the Chebyshev points of the second kind in $J$, and we study the rational interpolant (14) of the function $f: I \rightarrow \mathbb{C}$ between the $x_{k}$.

Let $w: \mathcal{D}_{1} \times \mathcal{D}_{1} \rightarrow \mathbb{C}$ be the analytic function of two variables [Kau-Kau]

$$
w(z, y):=\frac{z-y}{g(z)-g(y)} .
$$

In order to interpolate $f \circ g$ on the interval $J$, we write it as

$$
f(g(y))=\frac{f(g(y)) w(z, y)}{w(z, y)}
$$


where $(z, y) \in J \times J$, and we approximate both the numerator and the denominator of (15) by their interpolating polynomials between the Chebyshev points of second kind.

For the sake of interpolating $w(z, y)$ we freeze the variable $z$ and we construct the polynomial interpolating $w(z, y)$ between the $n+1$ Chebyshev points of the second kind $y_{k}=\cos \left(\frac{k \pi}{n}\right)$,

$$
P_{n}[w](z, y)=\sum_{k=0}^{n} w\left(z, y_{k}\right) L_{k}(y)=\frac{2^{n-1}}{n} L(y) \sum_{k=0}^{n} \frac{(-1)^{k}}{y-y_{k}} w\left(z, y_{k}\right) .
$$

When $z=y \neq y_{k}$ this yields

$$
D_{n}[w](y):=P_{n}[w](y, y)=\frac{2^{n-1}}{n} L(y) \sum_{k=0}^{n}{ }^{\prime \prime} \frac{(-1)^{k}}{g(y)-g\left(y_{k}\right)},
$$

and the approximated function now is $w(y, y):=\lim _{z \rightarrow y} w(z, y)=\frac{1}{g^{\prime}(y)} . D_{n}[w](y)$ (as a function of $y$ ) is no longer a polynomial. If we repeat the same operation for the numerator of the right hand side of (15), we obtain a function $N_{n}[(f \circ g) w](y)$ interpolating $f(g(y)) \frac{1}{g^{\prime}(y)}$ :

$$
N_{n}[(f \circ g) w](y):=\frac{2^{n-1}}{n} L(y) \sum_{k=0}^{n}{ }^{\prime \prime} \frac{(-1)^{k}}{g(y)-g\left(y_{k}\right)} f\left(g\left(y_{k}\right)\right) .
$$

By forming the quotient of (17) and (16), we obtain the following interpolant of $f \circ g$ :

$$
R_{n}[f \circ g](y):=\frac{N_{n}[(f \circ g) w](y)}{D_{n}[w](y)}=\frac{\sum_{k=0}^{n \prime \prime} \frac{(-1)^{k}}{g(y)-g\left(y_{k}\right)} f\left(g\left(y_{k}\right)\right)}{\sum_{k=0}^{n \prime \prime} \frac{(-1)^{k}}{g(y)-g\left(y_{k}\right)}} .
$$

With $x:=g(y)$ and $x_{k}:=g\left(y_{k}\right)$ this is precisely the rational function (14) for (conformally) transformed Chebyshev points. This new interpretation will allow us to prove the exponential convergence of (14) for analytic $f$.

The above construction can be performed for other points, such as transformed Chebyshev points of the first kind.

\section{Convergence}

Here we use results on the convergence of polynomials interpolating between Chebyshev points to prove the convergence of (14) between transformed Chebyshev points.

2.1. Convergence of the polynomial interpolating between Chebyshev points. The following two results can be found in [Boy, p. 139], and [Riv, p. 141], respectively.

Theorem 2. Let $\left\{a_{k}\right\}$ denote the exact coefficients in the Chebyshev series of $f$, that is,

$$
f(x)=\frac{a_{0}}{2}+\sum_{k=1}^{\infty} a_{k} T_{k}(x), \quad a_{k}:=\frac{2}{\pi} \int_{-1}^{1} f(x) T_{k}(x) \frac{d x}{\sqrt{1-x^{2}}}
$$


where the $T_{k}(x)$ are the Chebyshev polynomials of the first kind. Then the polynomial $P_{n}[f]$ interpolating $f$ between Chebyshev points of first or second kind satisfies

$$
\left|f(x)-P_{n}[f](x)\right| \leq 2 \sum_{k=n+1}^{\infty}\left|a_{k}\right|
$$

for all real $x$ and for all $n$.

The second theorem recalls that if $f$ is the restriction of an analytic function on $[-1,1]$, then the coefficients $a_{k}$ decay exponentially.

Theorem 3. If $f$ is analytic inside and on the ellipse $C_{\rho}, \rho>1$, with foci at \pm 1 , and if the sum of the major and minor axes of $C_{\rho}$ is equal to $2 \rho$, then

$$
\left|a_{k}\right| \leq \frac{2 M}{\rho^{k}},
$$

where $M:=\max _{z \in C_{\rho}}|f(z)|$.

For a function $f$ analytic in the interior and on an ellipse $C_{\rho}\left(\subset \mathcal{D}_{1}\right), \rho>1$, the preceding two theorems yield

$$
\left|f(x)-P_{n}[f](x)\right| \leq 4 M \sum_{k=n+1}^{\infty} \rho^{-k}=\frac{4 M}{\rho^{n}(\rho-1)},
$$

for all real $x$ and for all $n$.

2.2. Exponential convergence of the rational interpolant. We now come to the main result of the present work.

Theorem 4. Let $\mathcal{D}_{1}, \mathcal{D}_{2}$ be two domains of $\mathbb{C}$ containing $J=[-1,1]$, respectively $I(\in \mathbb{R})$, let $g$ be a conformal map $\mathcal{D}_{1} \rightarrow \mathcal{D}_{2}$ such that $g(J)=I$, and $f$ be a function $\mathcal{D}_{2} \rightarrow \mathbb{C}$ such that the composition $f \circ g: \mathcal{D}_{1} \rightarrow \mathbb{C}$ is analytic inside and on an ellipse $C_{\rho}\left(\subset \mathcal{D}_{1}\right), \rho>1$, with foci at \pm 1 and with the sum of its major and minor axes equal to $2 \rho$. Let $R_{n}[f](x) \equiv R_{n}[f \circ g](y), x=g(y)$, be the rational function (14) interpolating $f$ between the transformed Chebyshev points $x_{k}:=g\left(y_{k}\right)$. Then, for every $x \in[-1,1]$,

$$
\left|f(x)-R_{n}[f](x)\right|=\mathcal{O}\left(\rho^{-n}\right) .
$$

Proof. Let us apply the results of Section 2.1 to $P_{n}[w](z, y)$. For every fixed $z$, $P_{n}[w](z, y)$ is the polynomial interpolating $w$ between the Chebyshev points of second kind and, in view of the analyticity of $w: \mathcal{D}_{1} \times \mathcal{D}_{1} \rightarrow \mathbb{C}$, we have

$$
\left|w(z, y)-P_{n}[w](z, y)\right| \leq \frac{4 M_{1}(z)}{\tilde{\rho}^{n}(\tilde{\rho}-1)},
$$

where $M_{1}(z):=\max _{y \in C_{\tilde{\rho}}}|w(z, y)|$ and $C_{\tilde{\rho}}(\tilde{\rho} \geq \rho)$ is the largest among all ellipses $C_{\sigma}$ contained in $\mathcal{D}_{1}$ with foci at \pm 1 and with the sum of their axes equal to $2 \sigma$.

Defining $M_{1}:=\max _{z \in \mathcal{D}_{1}} M_{1}(z)=\max _{z \in \mathcal{D}_{1}, y \in C_{\tilde{\rho}}}|w(z, y)|$, this yields a bound for the error of the interpolant $D_{n}[w](y)$ :

$$
\left|w(y, y)-D_{n}[w](y)\right| \leq \frac{4 M_{1}}{\tilde{\rho}^{n}(\tilde{\rho}-1)} .
$$


Since $f \circ g$ is analytic inside and on the ellipse $C_{\rho}$, we similarly find for $N_{n}[(f \circ g) w](y)$

$$
\left|(f \circ g)(y) w(y, y)-N_{n}[(f \circ g) w](y)\right| \leq \frac{4 M_{2}}{\rho^{n}(\rho-1)},
$$

where $M_{2}:=\max _{z \in \mathcal{D}_{1}, y \in C_{\rho}}|(f \circ g)(y) w(z, y)|$.

Since $g$, conformal on $\mathcal{D}_{1}$, is continuous on $C_{\rho}$, we have $\min _{y \in C_{\rho}}|w(y, y)|>0$ and we can divide numerator and denominator by $w(y, y)$, which yields for the rational interpolant $R_{n}[f](x)$

$$
\begin{aligned}
R_{n}[f \circ g](y)=\frac{N_{n}[(f \circ g) w](y)}{D_{n}[w](y)} & =\frac{(f \circ g)(y) w(y, y)+\mathcal{O}\left(\rho^{-n}\right)}{w(y, y)+\mathcal{O}\left(\tilde{\rho}^{-n}\right)} \\
& =\frac{(f \circ g)(y)+\mathcal{O}\left(\rho^{-n}\right)}{1+\mathcal{O}\left(\tilde{\rho}^{-n}\right)} \\
& =(f \circ g)(y)+\mathcal{O}\left(\rho^{-n}\right) .
\end{aligned}
$$

\section{Numerical examples}

Here we displace the interpolation points with the mapping proposed by Kosloff and Tal-Ezer [Kos-Tal]

$$
g(y):=g(y, \alpha):=\frac{\arcsin (\alpha y)}{\arcsin (\alpha)}, \quad y \in[-1,1], \quad \alpha \in(0,1) .
$$

In the limit $\alpha=0$ the interpolation points $x_{k}:=g\left(y_{k}\right)$ remain the Chebyshev points (of second kind), whereas they become equidistant when $\alpha \rightarrow 1$. The derivative of $g$ has singularities at $\pm 1 / \alpha$. When $\alpha \rightarrow 1$ these singularities approach the interval of interpolation.

In order to quantify the displacement of the interpolation points, we give in Table 1 the quotient $\frac{h_{\min }}{h_{\max }}$, where $h_{\min }$ and $h_{\max }$ respectively denote the minimal and the maximal distances between the points. For the Chebyshev points the ratio

\begin{tabular}{|c|c|c|c|c|}
\hline \multirow[b]{3}{*}{$\alpha$} & \multicolumn{4}{|c|}{$\frac{h_{\min }}{h_{\max }}$} \\
\hline & \multicolumn{4}{|c|}{$n$} \\
\hline & 8 & 32 & 128 & 512 \\
\hline 0 & $1.99 \cdot 10^{-1}$ & $4.91 \cdot 10^{-2}$ & $1.23 \cdot 10^{-2}$ & $3.07 \cdot 10^{-3}$ \\
\hline 0.1 & $2.00 \cdot 10^{-1}$ & $4.94 \cdot 10^{-2}$ & $1.23 \cdot 10^{-2}$ & $3.08 \cdot 10^{-3}$ \\
\hline 0.5 & $2.26 \cdot 10^{-1}$ & $5.67 \cdot 10^{-2}$ & $1.42 \cdot 10^{-2}$ & $3.54 \cdot 10^{-3}$ \\
\hline 0.9 & $3.92 \cdot 10^{-1}$ & $1.11 \cdot 10^{-1}$ & $2.81 \cdot 10^{-2}$ & $7.04 \cdot 10^{-3}$ \\
\hline 0.94 & $4.64 \cdot 10^{-1}$ & $1.41 \cdot 10^{-1}$ & $3.59 \cdot 10^{-2}$ & $8.99 \cdot 10^{-3}$ \\
\hline 0.98 & $6.19 \cdot 10^{-1}$ & $2.34 \cdot 10^{-1}$ & $6.14 \cdot 10^{-2}$ & $1.54 \cdot 10^{-2}$ \\
\hline 0.99 & $7.07 \cdot 10^{-1}$ & $3.14 \cdot 10^{-1}$ & $8.64 \cdot 10^{-2}$ & $2.17 \cdot 10^{-2}$ \\
\hline 1 & 1 & 1 & 1 & 1 \\
\hline
\end{tabular}
is $\mathcal{O}(1 / n)$; for equidistant points it trivially equals 1 .

We have interpolated two test functions on the interval $[-1,1]$, both with the polynomial interpolating between the Chebyshev points of the second kind and with the rational function (14) for different $\alpha$. As a measure of the interpolation error, 
we have calculated the maximum among the absolute errors at the 1000 equidistant points $\widehat{x}_{k}:=-1+\frac{2 k+1}{1000}, k=0(1) 999$. The results are presented in Tables 2 and 3 .

In a further experiment we have approximated the first and the second derivatives of these two functions with a formula given in [Sch-Wer] and we have calculated the maximal absolute error at the interpolation points $x_{k}$. The corresponding results are presented in Tables 4, 5, 6, and 7 .

In all the tables, $\alpha=0$ refers to the polynomial (12) and the other $\alpha$ 's to the rational function (14). All the computations were done on a PowerMacintosh $8200 / 120$.

3.1. Interpolation. As the first example we chose Runge's classical example

$$
f(x):=\frac{1}{1+25 x^{2}} .
$$

Table 2 shows that the rational function yields better results than the polynomial as long as $\alpha$ is not too close to 1 . The exponential convergence reflects the analyticity of $f$.

The second example was

$$
k(x):=\sin (100 x) e^{-5 x} .
$$

$k$ oscillates a great deal on the interval $[-1,0]$; these oscillations are damped on the interval $[0,1] . \quad k$ is entire, and therefore the polynomial (12) interpolating between the Chebyshev points of the second kind as well as the rational function (14) converge exponentially toward $k$ (see Table 3 ).

TABLE 2. Interpolation error for $f$

\begin{tabular}{l|cccc|}
\multicolumn{5}{c}{$n$} \\
\cline { 2 - 5 }$\alpha$ & 8 & 32 & 128 & 512 \\
\hline 0 & $2.05 \cdot 10^{-1}$ & $1.62 \cdot 10^{-3}$ & $8.65 \cdot 10^{-12}$ & $3.33 \cdot 10^{-15}$ \\
0.1 & $2.04 \cdot 10^{-1}$ & $1.60 \cdot 10^{-3}$ & $8.28 \cdot 10^{-12}$ & $3.89 \cdot 10^{-15}$ \\
0.5 & $1.90 \cdot 10^{-1}$ & $1.21 \cdot 10^{-3}$ & $2.53 \cdot 10^{-12}$ & $3.22 \cdot 10^{-15}$ \\
0.9 & $1.35 \cdot 10^{-1}$ & $3.49 \cdot 10^{-4}$ & $1.55 \cdot 10^{-14}$ & $3.55 \cdot 10^{-15}$ \\
0.94 & $1.23 \cdot 10^{-1}$ & $2.43 \cdot 10^{-4}$ & $4.05 \cdot 10^{-15}$ & $3.11 \cdot 10^{-15}$ \\
0.98 & $1.03 \cdot 10^{-1}$ & $1.28 \cdot 10^{-4}$ & $1.11 \cdot 10^{-15}$ & $2.89 \cdot 10^{-15}$ \\
0.99 & $9.42 \cdot 10^{-2}$ & $9.34 \cdot 10^{-5}$ & $3.66 \cdot 10^{-13}$ & $2.00 \cdot 10^{-15}$ \\
1 & $7.34 \cdot 10^{-2}$ & $7.12 \cdot 10^{-5}$ & $7.17 \cdot 10^{-6}$ & $4.49 \cdot 10^{-7}$ \\
\hline
\end{tabular}

TABLE 3. Interpolation error for $k$

\begin{tabular}{l|cccc|}
\multicolumn{4}{c}{$n$} \\
\cline { 2 - 5 }$\alpha$ & 8 & 32 & 128 & 512 \\
\hline 0 & $2.21 \cdot 10^{2}$ & $1.59 \cdot 10^{2}$ & $1.06 \cdot 10^{-7}$ & $1.34 \cdot 10^{-12}$ \\
0.1 & $2.21 \cdot 10^{2}$ & $1.61 \cdot 10^{2}$ & $8.22 \cdot 10^{-8}$ & $1.28 \cdot 10^{-12}$ \\
0.5 & $1.87 \cdot 10^{2}$ & $1.81 \cdot 10^{2}$ & $5.42 \cdot 10^{-11}$ & $1.42 \cdot 10^{-12}$ \\
0.9 & $1.71 \cdot 10^{2}$ & $1.99 \cdot 10^{2}$ & $1.37 \cdot 10^{-12}$ & $1.47 \cdot 10^{-12}$ \\
0.94 & $2.00 \cdot 10^{2}$ & $1.91 \cdot 10^{2}$ & $1.11 \cdot 10^{-12}$ & $1.65 \cdot 10^{-12}$ \\
0.98 & $1.49 \cdot 10^{2}$ & $1.80 \cdot 10^{2}$ & $2.38 \cdot 10^{-9}$ & $1.26 \cdot 10^{-12}$ \\
0.99 & $1.89 \cdot 10^{2}$ & $1.99 \cdot 10^{2}$ & $3.42 \cdot 10^{-6}$ & $1.34 \cdot 10^{-12}$ \\
1 & $1.84 \cdot 10^{2}$ & $1.80 \cdot 10^{2}$ & $1.51 \cdot 10^{1}$ & $6.92 \cdot 10^{-1}$ \\
\hline
\end{tabular}


Tables 2 and 3 seem to demonstrate that when $n$ is large and increasing the optimal $\alpha$ moves closer to 1 . A possible explanation is the fact that, for constant $\alpha$, the points remain further from equidistant as $n$ increases (see the decrease of $\frac{h_{\min }}{h_{\max }}$ in Table 1), which results in not as good a sampling of the function. This phenomenon is even accentuated in the computation of derivatives, where the condition of the problem improves as the spacing of the point becomes more regular (see the tables in Section 3.2).

We also clearly see that the rational interpolant converges much more slowly for equidistant points $(\alpha=1)$ than for the others. We conjecture that the convergence of $R_{n}[f]$ is merely $\mathcal{O}\left(n^{-2}\right)$. However, for moderate $n$ (say $n \leq 32$ ) the regular sampling results in a better approximation.

3.2. Differentiation. We now recall a formula for the differentiation of rational functions. For that purpose, notice that every rational function $R_{n}[f]$ interpolating a function $f$ between the points $x_{k}$ can be written in its barycentric form

$$
R_{n}[f](x)=\frac{\sum_{k=0}^{n} \frac{u_{k}}{x-x_{k}} f_{k}}{\sum_{k=0}^{n} \frac{u_{k}}{x-x_{k}}}
$$

[Ber-Mit], where $f_{k}:=f\left(x_{k}\right)$ and where the $u_{k}$ are the barycentric weights of the rational interpolant.

In order to calculate the derivatives of a rational function written in such a form we use the following results of Schneider and Werner [Sch-Wer].

Proposition 1. Let $R_{n}[f]$ be a rational function given in its barycentric form (24), with $u_{k} \neq 0, k=0(1) n$. Assume that $\xi$ is not a pole of $R_{n}[f]$; then for $\xi \neq x_{k}, k=$ $0(1) n$,

$$
\frac{R_{n}^{(m)}[f](\xi)}{m !}=\sum_{k=0}^{n} \frac{u_{k}}{\xi-x_{k}} R_{n}[f]\left[(\xi)^{m}, x_{k}\right] / \sum_{k=0}^{n} \frac{u_{k}}{\xi-x_{k}}, \quad m \geq 0
$$

and

$$
\frac{R_{n}^{(m)}[f]\left(x_{i}\right)}{m !}=-\left(\sum_{k=0, k \neq i}^{n} u_{k} R_{n}[f]\left[\left(x_{i}\right)^{m}, x_{k}\right]\right) / u_{i}, \quad 0 \leq i \leq n, \quad m \geq 1 .
$$

The notation $(\xi)^{m}$ is used here to indicate the $m$-fold argument $\xi, \xi, \ldots, \xi$, and $R_{n}[f]\left[x_{0}, \ldots, x_{k}\right]$ denotes the $k$-th order divided difference of $R_{n}[f]$ with

$$
R_{n}[f][\underbrace{\xi, \ldots, \xi}_{m+1}]=\frac{R_{n}^{(m)}[f](\xi)}{m !} .
$$

Making use of formula (26), we can compute the first two derivatives of the function (24) at the interpolation points $x_{i}$ by constructing the differentiation matrices $D^{(1)}$ 
and $D^{(2)}$, whose entries are given by

$$
D_{i j}^{(1)}= \begin{cases}\frac{u_{j}}{u_{i}} \frac{1}{x_{i}-x_{j}}, & i \neq j \\ -\sum_{k=0, k \neq i} \frac{u_{k}}{u_{i}} \frac{1}{x_{i}-x_{k}}, & i=j\end{cases}
$$

respectively

$$
D_{i j}^{(2)}= \begin{cases}2 D_{i j}^{(1)}\left(D_{i i}^{(1)}-\frac{1}{x_{i}-x_{j}}\right), & i \neq j, \\ 2\left(D_{i i}^{(1)}\right)^{2}+2 \sum_{k=0, k \neq i}^{n} D_{i k}^{(1)} \frac{1}{x_{i}-x_{k}}, & i=j .\end{cases}
$$

Indeed, by multiplying the vector $\mathbf{f}$ of the values $f_{k}$ of $f$ at the interpolation points by the matrix $D^{(1)}$ we obtain the vector $\mathbf{f}^{\prime}$ of the first derivative of the rational function (24) at the same points: $\mathbf{f}^{\prime}=D^{(1)} \mathbf{f}$. Doing the same for the matrix $D^{(2)}$, we obtain the second derivative at the interpolation points, $\mathbf{f}^{\prime \prime}:=D^{(2)} \mathbf{f}$. Higher derivatives can be similarly computed with formula (26).

3.2.1. First derivative. The derivative of the functions (22) and (23) will now be approximated at the interpolation points. For the polynomial interpolating between the Chebyshev points $y_{k}=\cos \left(\frac{k \pi}{n}\right)$, we use the formulas for the differentiation matrix $D^{(1)}$ given in books on spectral methods like [Boy],

$$
\begin{array}{ll}
D_{i j}^{(1)}=\frac{c_{i}}{c_{j}} \frac{(-1)^{i+j}}{y_{i}-y_{j}}, & (i \neq j), \\
D_{j j}^{(1)}=-\frac{y_{j}}{2\left(1-y_{j}^{2}\right)}, & (j \neq 0, n), \quad D_{00}^{(1)}=\frac{2 n^{2}+1}{6}=-D_{n n}^{(1)},
\end{array}
$$

with $c_{0}=c_{n}=2$ and $c_{k}=1$ for $k=0(1) n$. For the rational function, we use (27). The approximation results for $f^{\prime}$ are presented in Table 4.

From 128 to 512 points the approximation with the interpolating polynomial does not improve. As explained in [Bal-Ber1], the main reason for this is believed to be the extreme precision of the elements $D_{00}^{(1)}$ and $D_{n n}^{(1)}$ as compared with the other elements of the differentiation matrix.

TABle 4. Approximation error for $f^{\prime}$

\begin{tabular}{l|cccc|}
\multicolumn{5}{c}{$n$} \\
\cline { 2 - 5 }$\alpha$ & 8 & 32 & 128 & 512 \\
\hline 0 & $1.37 \cdot 10^{0}$ & $5.44 \cdot 10^{-2}$ & $1.13 \cdot 10^{-9}$ & $6.86 \cdot 10^{-9}$ \\
0.1 & $1.37 \cdot 10^{0}$ & $5.39 \cdot 10^{-2}$ & $1.08 \cdot 10^{-9}$ & $1.20 \cdot 10^{-12}$ \\
0.5 & $1.36 \cdot 10^{0}$ & $4.19 \cdot 10^{-2}$ & $3.47 \cdot 10^{-10}$ & $2.15 \cdot 10^{-12}$ \\
0.9 & $1.25 \cdot 10^{0}$ & $1.38 \cdot 10^{-2}$ & $2.47 \cdot 10^{-12}$ & $1.82 \cdot 10^{-12}$ \\
0.94 & $1.21 \cdot 10^{0}$ & $1.01 \cdot 10^{-2}$ & $6.01 \cdot 10^{-13}$ & $2.91 \cdot 10^{-12}$ \\
0.98 & $1.14 \cdot 10^{0}$ & $5.80 \cdot 10^{-3}$ & $2.89 \cdot 10^{-14}$ & $5.71 \cdot 10^{-13}$ \\
0.99 & $1.10 \cdot 10^{0}$ & $4.37 \cdot 10^{-3}$ & $6.79 \cdot 10^{-11}$ & $7.69 \cdot 10^{-13}$ \\
1 & $9.78 \cdot 10^{-1}$ & $3.59 \cdot 10^{-3}$ & $1.44 \cdot 10^{-3}$ & $3.61 \cdot 10^{-4}$ \\
\hline
\end{tabular}


TABle 5. Approximation error for $k^{\prime}$

\begin{tabular}{l|cccc|}
\multicolumn{5}{c}{$n$} \\
\cline { 2 - 5 }$\alpha$ & 8 & 32 & 128 & 512 \\
\hline 0 & $1.13 \cdot 10^{4}$ & $1.31 \cdot 10^{4}$ & $2.51 \cdot 10^{-5}$ & $8.30 \cdot 10^{-9}$ \\
0.1 & $1.13 \cdot 10^{4}$ & $1.31 \cdot 10^{4}$ & $2.01 \cdot 10^{-5}$ & $6.07 \cdot 10^{-8}$ \\
0.5 & $1.26 \cdot 10^{4}$ & $1.24 \cdot 10^{4}$ & $1.28 \cdot 10^{-8}$ & $5.15 \cdot 10^{-8}$ \\
0.9 & $1.31 \cdot 10^{4}$ & $1.31 \cdot 10^{4}$ & $1.73 \cdot 10^{-9}$ & $5.16 \cdot 10^{-8}$ \\
0.94 & $1.22 \cdot 10^{4}$ & $1.43 \cdot 10^{4}$ & $7.80 \cdot 10^{-10}$ & $2.95 \cdot 10^{-8}$ \\
0.98 & $1.37 \cdot 10^{4}$ & $1.39 \cdot 10^{4}$ & $4.58 \cdot 10^{-7}$ & $1.70 \cdot 10^{-8}$ \\
0.99 & $1.26 \cdot 10^{4}$ & $1.45 \cdot 10^{4}$ & $2.23 \cdot 10^{-3}$ & $1.29 \cdot 10^{-8}$ \\
1 & $1.27 \cdot 10^{4}$ & $1.28 \cdot 10^{4}$ & $3.91 \cdot 10^{3}$ & $8.63 \cdot 10^{2}$ \\
\hline
\end{tabular}

As in the case of the interpolation, the approximation with the rational function (14) behaves like the approximation with the polynomial (12) and is even better in most cases.

The derivative of the rational function interpolating between equidistant points $(\alpha=1)$ converges toward the derivative of $f$ at the rate $\mathcal{O}\left(n^{-1}\right)$, losing a power of $n$ in the course of the differentiation.

The results for the derivative

$$
k^{\prime}(x)=5 e^{-5 x}(20 \cos (100 x)-\sin (100 x))
$$

of the function (23) are comparable with those for (22). The approximation is not as good for small $n$, however, because $k^{\prime}$ oscillates a great deal on the interval $[-1,0]$ (see Table 5$)$.

3.2.2. Second derivative. We use a similar procedure to compute the error in approximating

$$
f^{\prime \prime}(x)=50\left(75 x^{2}-1\right) /\left(1+25 x^{2}\right)^{3} .
$$

We calculate the differentiation matrix $D^{(2)}$ by the formulas (28) for the rational function (14), but we make use of the relation $D^{(2)}=\left[D^{(1)}\right]^{2}$ for the polynomial interpolating between the Chebyshev points [Boy]. Again, the error with the polynomial (12) does not improve when the number of points increases from 128 to 512 . In fact it becomes even worse, which can be explained in the same way as for the first derivative [Bal-Ber1].

The error does not always improve for the rational function (14) either, but the worsening effect is attenuated. For $\alpha=1$, the approximation of the second derivative is $\mathcal{O}(1)$ (see Table 6 ).

The accuracy of the approximation of

$$
k^{\prime \prime}(x)=-25 e^{-5 x}(40 \cos (100 x)+399 \sin (100 x))
$$

by the second derivative of (14) loses at least one power of $n$. As $\alpha \rightarrow 1$, the approximation becomes disastrous. However, as long as $\alpha$ is not too close to 1 , the approximation remains accurate for sufficiently large $n$. For $n=128$ the improvement with respect to the polynomial interpolating between the Chebyshev points is remarkable (see Table 7). 
TABLE 6. Approximation error for $f^{\prime \prime}$

\begin{tabular}{l|cccc|}
\multicolumn{5}{c}{$n$} \\
\cline { 2 - 5 }$\alpha$ & 8 & 32 & 128 & 512 \\
\hline 0 & $5.29 \cdot 10^{1}$ & $2.85 \cdot 10^{1}$ & $9.33 \cdot 10^{-6}$ & $6.86 \cdot 10^{-4}$ \\
0.1 & $5.26 \cdot 10^{1}$ & $2.81 \cdot 10^{1}$ & $9.08 \cdot 10^{-6}$ & $1.15 \cdot 10^{-6}$ \\
0.5 & $4.65 \cdot 10^{1}$ & $2.00 \cdot 10^{1}$ & $2.64 \cdot 10^{-6}$ & $3.28 \cdot 10^{-7}$ \\
0.9 & $2.77 \cdot 10^{1}$ & $3.99 \cdot 10^{0}$ & $1.17 \cdot 10^{-8}$ & $8.01 \cdot 10^{-8}$ \\
0.94 & $2.63 \cdot 10^{1}$ & $2.38 \cdot 10^{0}$ & $2.33 \cdot 10^{-9}$ & $1.73 \cdot 10^{-7}$ \\
0.98 & $2.40 \cdot 10^{1}$ & $8.71 \cdot 10^{-1}$ & $6.97 \cdot 10^{-11}$ & $2.61 \cdot 10^{-8}$ \\
0.99 & $2.30 \cdot 10^{1}$ & $4.95 \cdot 10^{-1}$ & $1.17 \cdot 10^{-7}$ & $3.11 \cdot 10^{-9}$ \\
1 & $2.01 \cdot 10^{1}$ & $1.18 \cdot 10^{-1}$ & $1.95 \cdot 10^{-1}$ & $1.97 \cdot 10^{-1}$ \\
\hline
\end{tabular}

TABLE 7. Approximation error for $k^{\prime \prime}$

\begin{tabular}{l|cccc|}
\multicolumn{5}{c}{$n$} \\
\cline { 2 - 5 }$\alpha$ & 8 & 32 & 128 & 512 \\
\hline 0 & $9.30 \cdot 10^{5}$ & $2.71 \cdot 10^{6}$ & $2.74 \cdot 10^{-1}$ & $1.21 \cdot 10^{-3}$ \\
0.1 & $9.20 \cdot 10^{5}$ & $2.60 \cdot 10^{6}$ & $2.19 \cdot 10^{-1}$ & $4.58 \cdot 10^{-3}$ \\
0.5 & $8.74 \cdot 10^{5}$ & $5.86 \cdot 10^{5}$ & $1.26 \cdot 10^{-4}$ & $1.95 \cdot 10^{-3}$ \\
0.9 & $8.83 \cdot 10^{5}$ & $4.67 \cdot 10^{6}$ & $3.09 \cdot 10^{-6}$ & $2.38 \cdot 10^{-3}$ \\
0.94 & $8.74 \cdot 10^{5}$ & $2.81 \cdot 10^{6}$ & $9.65 \cdot 10^{-7}$ & $6.04 \cdot 10^{-4}$ \\
0.98 & $8.84 \cdot 10^{5}$ & $8.00 \cdot 10^{5}$ & $8.48 \cdot 10^{-4}$ & $3.76 \cdot 10^{-4}$ \\
0.99 & $8.78 \cdot 10^{5}$ & $1.04 \cdot 10^{6}$ & $5.00 \cdot 10^{0}$ & $2.45 \cdot 10^{-4}$ \\
1 & $8.78 \cdot 10^{5}$ & $8.78 \cdot 10^{5}$ & $3.74 \cdot 10^{5}$ & $5.37 \cdot 10^{5}$ \\
\hline
\end{tabular}

\section{CONCLUSion}

We have shown the exponential convergence of the rational function (14) presented in [Ber1] for conformally transformed Chebyshev points. Making use of the formula given in [Sch-Wer], we have also calculated its first two derivatives at the interpolation points, which in many instances are significantly more precise than those of the polynomial interpolating between Chebyshev points.

In a future work, we intend to use these rational interpolants for solving partial differential equations [Bal-Ber2].

\section{ACKNowledgments}

The authors wish to thank the unknown referee whose comments have improved the text of the present paper.

\section{REFERENCES}

[Bal-Ber1] Baltensperger, R. and Berrut, J.-P.: The errors in calculating the pseudospectral differentiation matrices for Chebyshev-Gauss-Lobatto points, to appear in Comput. Math. Applic.

[Bal-Ber2] Baltensperger, R. and Berrut, J.-P.: The linear rational collocation method, submitted for publication.

[Ber1] Berrut, J.-P.: Rational functions for guaranteed and experimentally well-conditioned global interpolation, Comput. Math. Applic., 15, 1-16 (1988). MR 89b:65029

[Ber2] Berrut, J.-P.: Barycentric formulae for cardinal (SINC-) interpolants, Numer. Math., 54, 703-718 (1989) (Erratum 55, 747 (1989)). MR 90d:65025a,b 
1120 RICHARD BALTENSPERGER, JEAN-PAUL BERRUT, AND BENJAMIN NOËL

[Ber-Mit] Berrut, J.-P. and Mittelmann, H. D.: Lebesgue constant minimizing linear rational interpolation of continuous functions over the interval, Comput. Math. Applic., 33, 77-86 (1997). MR 98c:41015

[Boy] Boyd, J. P.: Chebyshev and Fourier Spectral Methods, Springer Verlag, BerlinHeidelberg-New York (1989).

[Hen] Henrici, P.: Essentials of Numerical Analysis with Pocket Calculator Demonstrations, Wiley, New York (1982). MR 83h:65002

[Kau-Kau] Kaup, L. and Kaup, B.: Holomorphic Functions of Several Variables, de Gruyter, Berlin-New York (1983). MR 85k:32001

[Kos-Tal] Kosloff, D. and Tal-Ezer, H.: A modified Chebyshev pseudospectral method with an $\mathcal{O}\left(N^{-1}\right)$ time step restriction, J. Comput. Phys., 104, 457-469 (1993). MR 93k:65080

[Riv] Rivlin, T. J.: The Chebyshev Polynomials, Wiley, New York (1974). MR 56:9142

[Sal] Salzer, H. E.: Lagrangian interpolation at the Chebyshev points $x_{n, \nu}=$ $\cos (\nu \pi / n), \quad \nu=0(1) n$; some unnoted advantages, The Computer J., 15, 156-159 (1972). MR 47:4414

[Sch-Wer] Schneider, C. and Werner, W.: Some new aspects of rational interpolation, Math. Comp., 47, 285-299 (1986). MR 87k:65012

[Sch] Schwarz, H. R.: Numerische Mathematik, 2te Aufl., Teubner, Stuttgart (1988); English translation: Numerical Analysis, A Comprehensive Introduction, Wiley, New-York (1989). MR 92a:65008; MR 90g:65003

[Sto] Stoer, J.: Einführung in die Numerische Mathematik I, 4te Aufl., Springer, Berlin (1983). MR 83d:65003 (3rd ed.)

Institut de Mathématiques, Université de Fribourg, Pérolles, CH-1700 Fribourg, SWITZERLAND

E-mail address: richard.baltensperger@unifr.ch

E-mail address: jean-paul.berrut@unifr.ch 